\title{
Cannabisin B induces autophagic cell death by inhibiting the AKT/mTOR pathway and S phase cell cycle arrest in HepG2 cells
}

\author{
Tianpeng Chen ${ }^{\mathrm{a}, \mathrm{b}}$, Jianxiong Hao ${ }^{\mathrm{c}}$, Jinfeng $\mathrm{He}^{\mathrm{b}}$, Jianchun Zhang ${ }^{\mathrm{b}}$, Yingcong Li ${ }^{\mathrm{d}}$, Rui Liu ${ }^{\mathrm{e}}$, Lite $\mathrm{Li}^{\mathrm{e}, *}$ \\ ${ }^{a}$ College of Engineering, Peking University, Beijing 100871, China \\ ${ }^{\mathrm{b}}$ The Research Center of China-Hemp Materials, The Quartermaster Research Institute, The General Logistic Department, Beijing 100082, China \\ ${ }^{\mathrm{c}}$ Hebei University of Science and Technology, Shijiazhuang, Hebei 050054, China \\ ${ }^{\mathrm{d}}$ Institute of Psychology, Chinese Academy of Sciences, Beijing 100083, China \\ ${ }^{\mathrm{e}}$ College of Food Science and Nutritional Engineering, China Agricultural University, Beijing 100083, China
}

\section{A R T I C L E I N F O}

\section{Article history:}

Received 6 July 2012

Received in revised form 9 November 2012

Accepted 21 November 2012

Available online 5 December 2012

\section{Keywords:}

Cannabisin B

Antiproliferative

Autophagy

AKT

mTOR

Cell cycle arrest

\begin{abstract}
A B S T R A C T
This study investigates the anticancer properties of cannabisin B, purified from hempseed hull, in HepG2 human hepatoblastoma cells. The results indicate that cannabisin B significantly inhibited cell proliferation by inducing autophagic cell death rather than typical apoptosis. Cell viability transiently increased upon the addition of a low concentration of cannabisin B but decreased upon the addition of high concentrations. Cannabisin B-induced changes in cell viability were completely inhibited by pre-treatment with 3-methyladenine (3-MA), indicating that the induction of autophagy by cannabisin B caused cell death. Additionally, cannabisin B induced S phase cell cycle arrest in a dose-dependent manner. Moreover, cannabisin B was found to inhibit survival signaling by blocking the activation of AKT and down-stream targets of the mammalian target of rapamycin (mTOR). These findings suggest that cannabisin B possesses considerable antiproliferative activity and that it may be utilised as a promising chemopreventive agent against hepatoblastoma disease.
\end{abstract}

() 2012 Elsevier Ltd. All rights reserved.

\section{Introduction}

Autophagy, which is described as the mass degradation of cellular components, is an essential and homeostatic process that enables cells to adapt to adverse situations, such as nutrient deprivation and invasion by microorganisms (Levine, Mizushima, \& Virgin, 2011). Autophagic cell death (also known as type II programmed cell death) plays an important role in metabolism and stress responses as well as human diseases, including neurodegeneration, inflammation, muscular disorders, and resistance to pathogens (Mizushima \& Levine, 2010). In eukaryotic cells, autophagy commonly occurs within a double-membraned vesicle, which is then hydrolysed by the lysosome. Although the molecular signaling pathways are poorly understood, several molecules have been discovered to regulate autophagy, including members of the BECN1, DAPK (death-associated protein kinase), DRP1 (death-associated related protein kinase 1), MAPK (mitogen-activated kinase), and PI3K-AKT-mTOR (phosphatidylinositol 3-kinase/AKT/mammalian target of rapamycin) pathways (Kelekar, 2008; Todde, Veenhuis, \& van der Klei, 2009). Recent reports indicate that

* Corresponding author. Address: Post Box 40, China Agricultural University, No.17 Qinghua Donglu, Haidian District, Beijing 100083, China. Tel.: +86 10 62736442; fax: +86 1062737331 .

E-mail address: llt@cau.edu.cn (L. Li). autophagy mediates chemotherapy-induced cell death in cancer, functioning as a suppressor in early tumorigenesis but a stimulator in established tumors (Notte, Leclere, \& Michiels, 2011).

Hepatoblastoma is a frequent liver tumor in Western countries often occurring in infants and children. The disease is most commonly diagnosed during a child's first three years and they are usually present with an abdominal mass (De Ioris et al., 2008). Hepatoblastomas originate from immature liver precursor cells. People with familial adenomatous polyposis (FAP), a syndrome of early-onset colonic polyps and adenocarcinoma, frequently develop hepatoblastomas (Hirschman, Pollock, \& Tomlinson, 2005). Currently, there is no effective drug treatment for hepatoblastoma except for surgical resection. If metastases occur, the patient's condition is usually out of control. Therefore, the development of new agents, particularly phytochemicals from plants, is important for the treatment of this liver tumor.

Cannabisin B, a lignanamide from hempseed, was first identified in 1992 (Sakakibara, Ikeya, Hayashi, \& Mitsuhashi, 1992). The bioactivity of this hempseed secondary metabolite remained unevaluated until recently, when we demonstrated that cannabisin B functions predominantly as an antioxidant in hempseed hull (Chen et al., 2012). Several reports have indicated that the antioxidant substances in plants exhibit antiproliferative activity in cancer cells (He \& Liu, 2008; Prasad et al., 2010; Yang, Liu, \& Halim, 2009). Thus, the objectives of the current study were (1) to 
examine the antiproliferative activity of cannabisin B and the crude extract of hempseed hull in HepG2 cells and (2) to investigate the autophagy-related molecules associated with the cell death signal transduction pathway.

\section{Materials and methods}

\subsection{Materials}

Dulbecco's modified Eagle's medium (DMEM) and fetal bovine serum (FBS) were purchased from GIBCO, NY, USA. The cell counting kit-8, cell cycle analysis kit, annexin V-FITC apoptosis detection kit, and DNA ladder extraction kit with spin columns were obtained from Beyotime Institute of Biotechnology, Jiangsu China. Monodansylcadaverine (MDC), 3-methyladenine (3-MA), and Hoechst 33258 were purchased from Sigma-Aldrich, USA. Acetonitrile (HPLC grade) was purchased from Fisher, USA.

\subsection{Sample preparation}

The hempseed hull was separated from the hull using a laboratory huller machine (model JLGJ4.5, Zhejiang Liangyi Co., China) and milled in a high speed grinder (FW135, Tianjin Taisite Instrument Co., China) then through $380 \mu \mathrm{m}$ meshes. The hull powder $(1.0 \mathrm{~kg}$ ) was extracted using $60 \%$ EtOH water solution in an ultrasonic bath (KQ-100E, Jiangsu Kunshan Ultrasonic Instrument Co., China) at $40 \mathrm{kHz}(20 \mathrm{~L} \times 30 \mathrm{~min})$. The extract solution was then vacuum-evaporated to remove the EtOH, purified in a HPD-600 resin column, and eluted with water and $20 \% \mathrm{EtOH}$. Eluent of $40 \%$ EtOH was collected and lyophilized to produce the crude extract ( $1.0 \mathrm{~g}$, yellow powder). The crude extract was then applied to a Sephadex LH-20 column and eluted using 20\%, 40\%, and 60\% $\mathrm{MeOH}$. The collected $60 \% \mathrm{MeOH}$ eluent was finally purified by HPLC to obtain cannabisin B (70 mg).

\subsection{HPLC measurement}

Purification and quantification were conducted using a HPLC system equipped with a DAD (model LC-20A, Shimadzu, Japan). The sample was dissolved in $60 \% \mathrm{MeOH}$ and filtered through a $0.45 \mathrm{~nm}$ filter. Column chromatography was performed on a C18 column $(250 \mathrm{~mm} \times 4.6 \mathrm{~mm}$ i.d., $5 \mu \mathrm{m}$, model ST, Techmate Co., China) at $35^{\circ} \mathrm{C}$ with a monitoring wavelength of $335 \mathrm{~nm}$. The binary mobile phase consisted of (A) water- $0.1 \%$ acetic acid and (B) acetonitrile- $0.1 \%$ acetic acid. The gradient elution (at a flow rate of $1 \mathrm{ml} / \mathrm{min}$ ) was as follows: $0-20 \mathrm{~min}, 10-30 \% \mathrm{~B}$; $20-30 \mathrm{~min}$, $30 \%$ B isocratic; $30-32 \mathrm{~min}, 10-30 \% \mathrm{~B}$; $32-40 \mathrm{~min}, 10 \% \mathrm{~B}$ isocratic.

\subsection{Cell culture and treatment}

The HepG2 cell line was purchased from Shanghai Institute of Biochemistry and Cell Biology (SIBCB). Cells were grown in 90\% DMEM supplemented with $10 \%$ fetal bovine serum (FBS), 100 units $/ \mathrm{ml}$ penicillin, and $100 \mu \mathrm{g} / \mathrm{ml}$ streptomycin. The cell cultures were maintained at $37{ }^{\circ} \mathrm{C}$ in a humidified atmosphere of $5 \%$ $\mathrm{CO}_{2}$. The medium was changed every other day, and the cells were subcultured at $1: 2$ every 3 days. The cells were then plated at an appropriate density according to each experimental scale.

\subsection{Cell proliferation assay}

WST- 8 was employed to analyze cell proliferation because it is reduced the soluble formazan by mitochondrial dehydrogenase and exhibits little toxicity in cells. Cell viability was determined using the WST-8 assay kit (Beyotime Institute of Biotechnology,
China) according to the manufacturer's instructions. Briefly, $1 \times 10^{4}$ cells/well were seeded in a 96-well flat-bottomed plate, grown at $37^{\circ} \mathrm{C}$ for $24 \mathrm{~h}$, washed with PBS, and then treated with various concentrations $(84,167,251,335$, or $503 \mu \mathrm{M})$ of cannabisin B extract dissolved in DMEM for 24,48 , or $72 \mathrm{~h}$. Cells incubated in DMEM without cannabisin B extract served as the control. WST8 dye was added to each well, and the cells were incubated at $37^{\circ} \mathrm{C}$ for $2 \mathrm{~h}$. The absorbance was finally determined at both 450 and $630 \mathrm{~nm}$ using a microplate reader (Bio-Rad 550, Japan). Values were expressed as the percentage of control cells.

\subsection{Cell cycle analysis}

The cells were seeded into a 6-well plate, incubated for $24 \mathrm{~h}$, and then treated with concentrations of 167,335 , or $503 \mu \mathrm{M}$ cannabisin B for $24 \mathrm{~h}$. The cells were then fixed with $70 \%$ ice-cold methanol and transferred to the freezer until analysis. Upon analysis, the cells were washed with PBS and then stained with $50 \mu \mathrm{g} / \mathrm{ml}$ propidium iodide in the presence of $25 \mu \mathrm{g} / \mathrm{ml}$ RNase A at $37^{\circ} \mathrm{C}$ for $30 \mathrm{~min}$. The cell cycle distribution of 10,000 cells was recorded by a flow cytometer (BD FACS Calibur), and the percentage of cells in the G0/G1, S, and G2/M phases was analyzed with ModFit software.

\subsection{Morphological detection of HepG2 cells}

Cell morphology and nuclear morphology were both assessed with a fluorescent microscope (Olympus IX71, Japan). The cells were treated with 167,335 , or $503 \mu \mathrm{M}$ for $24 \mathrm{~h}$ and remained untreated cells as controls. The cells were observed after being stained with $10 \mu \mathrm{g} / \mathrm{ml}$ Hoechst 33258 for $15 \mathrm{~min}$. The cells were also stained with MDC in the same manner as described above.

\subsection{Analysis of apoptosis by Annexin V-FITC binding}

The cells were seeded and treated in the same manner as described for cell cycle analysis. The cells were then collected, washed twice with PBS, and stained using the annexin V-FITC apoptosis detection kit (Beyotime Institute of Biotechnology, China) as described by the manufacturer's instruction (Jiang, Zu, Fu, Zhang, \& Efferth, 2008). The fraction of the cell population that appeared in each quadrant was analyzed using quadrant statistics. The lower left quadrant contained intact cells, the lower right quadrant contained apoptotic cells, and the upper right quadrant contained necrotic or post-apoptotic cells. The results were expressed as the percentage of the total number of cells.

\subsection{DNA fragmentation analysis}

Fragmented DNA was isolated using the DNA extraction kit (Beyotime Institute of Biotechnology, China) according to the manufacturer's instructions. The samples were treated in the same manner as described for cell cycle analysis. The eluents containing DNA pellets were electrophoresed on a $1.0 \%$ agarose gel at $80 \mathrm{~V}$ for $1.5 \mathrm{~h}$. The gel was examined and photographed using an ultraviolet gel imaging system.

\subsection{Transmission Electron Microscopy (TEM)}

The HepG2 cells were treated with cannabisin B for $24 \mathrm{~h}$ and then collected and centrifuged at $1000 \mathrm{~g}$ for $5 \mathrm{~min}$. The fixation procedures and the manufacturing of resin samples were manipulated according to a previous report (Lin et al., 2007). The thin slice was observed with a Hitachi 7650 electron microscope (Tokyo, Japan). 


\subsection{Western blot analysis}

After treatment with cannabisin B, cells in $6 \mathrm{~cm}$ plate were carefully rinsed twice with PBS. After lysis in cold radioimmunoprecipitation assay buffer containing protease inhibitors, the cells were scraped and centrifuged at $13000 \mathrm{~g}$ for $10 \mathrm{~min}$ at $4{ }^{\circ} \mathrm{C}$. The liquid supernatants were then collected and mixed with electrophoresis loading buffer. The protein samples were normalized by loading equal amounts of total protein lysates. Protein samples were separated by SDS-PAGE, transferred onto a nitrocellulose membrane, and then probed with the primary antibody, which was followed by the addition of the secondary antibody conjugated with alkaline phosphatase. The bands were finally detected using the Alkaline Phosphatase Color Development Kit (Beyotime Institute of Biotechnology, China). Antibodies specific for $\beta$-actin, LC-3B, Akt, phosphoAkt (Ser473), mTOR, and phospho-mTOR were purchased from Beyotime Institute of Biotechnology (Jiangsu, China). The assay was repeated at least three times and the clearest one would be imaged for analysis.

\subsection{Statistical analysis}

Statistical analyses were performed using the SPSS 16.0 software package (SPSS Inc., Chicago, IL, USA). All experimental measurements were conducted in at least triplicate, and the data were expressed as the mean \pm standard deviation (SD). Differences between samples were evaluated by using an analysis of variance (ANOVA) and Duncan's multiple comparison method. Results were considered statistically significant if $p<0.05$.

\section{Results}

\subsection{Inhibition of Cannabisin B and crude extract on HepG2 cells}

In our previous study, we confirmed that cannabisin B from hempseed hull extract possesses predominantly antioxidant activities (Chen et al., 2012). In this report, cannabisin B was separated and purified by solvent extraction, macroporous resin absorption,
LH-20 gel chromatography, and HPLC (Fig. 1) in order to be evaluated for antiproliferative activity in cancer cells.

The inhibitory effects of cannabisin B and crude extract from resin column purification on HepG2 cells are shown in Fig. 2A. Each sample significantly inhibited the growth of HepG2 cells in a concentration-dependent manner, with the exception being cannabisin B treatment for $24 \mathrm{~h}$. Cannabisin B also exhibited a time-dependent antiproliferative activity when the concentration was greater than $251 \mu \mathrm{M}$. Although the crude extract exhibited a stronger inhibition activity, the treated cells shape indicated it might be attributed to necrosis (data not showed).

Visible light pictures of the control cells and the cells treated with different doses of cannabisin B were taken to assess cell morphology (Fig. 2B). The cells shrunk and became round upon treatment with cannabisin $B$, and the samples that received $503 \mu \mathrm{M}$ cannabisin B exhibited obvious degradation compared with the control cells. These changes in cell morphology occurred in a dose-dependent manner. The results indicated that both cannabisin B and crude extract can inhibit the growth of HepG2 cells and that cannabisin B exerted its effects in a dose- and timedependent manner.

\subsection{Cannabisin B-induced S phase arrest in HepG2 cells}

The effect of cannabisin B on cell cycle profile was analyzed by flow cytometry. The distribution of cells in different phases of the cell cycle is shown in Fig. 3. A significant and dose-dependent accumulation of cells was observed in the S phase, increasing from $21 \%$ in the control cells to $43.84 \%$ in the cells treated with $503 \mu \mathrm{M}$ cannabisin B. No obvious change was observed in the G2/M phase. The results indicated that cannabisin B could arrest HepG2 cells in $S$ phase and subsequently block cell growth.

\subsection{Cannabisin B can not induce apoptosis in HepG2 Cells}

Apoptosis is a programmed death process that is defined by a number of morphological and biochemical changes, including cell shrinkage, chromatin condensation, DNA fragmentation, and the

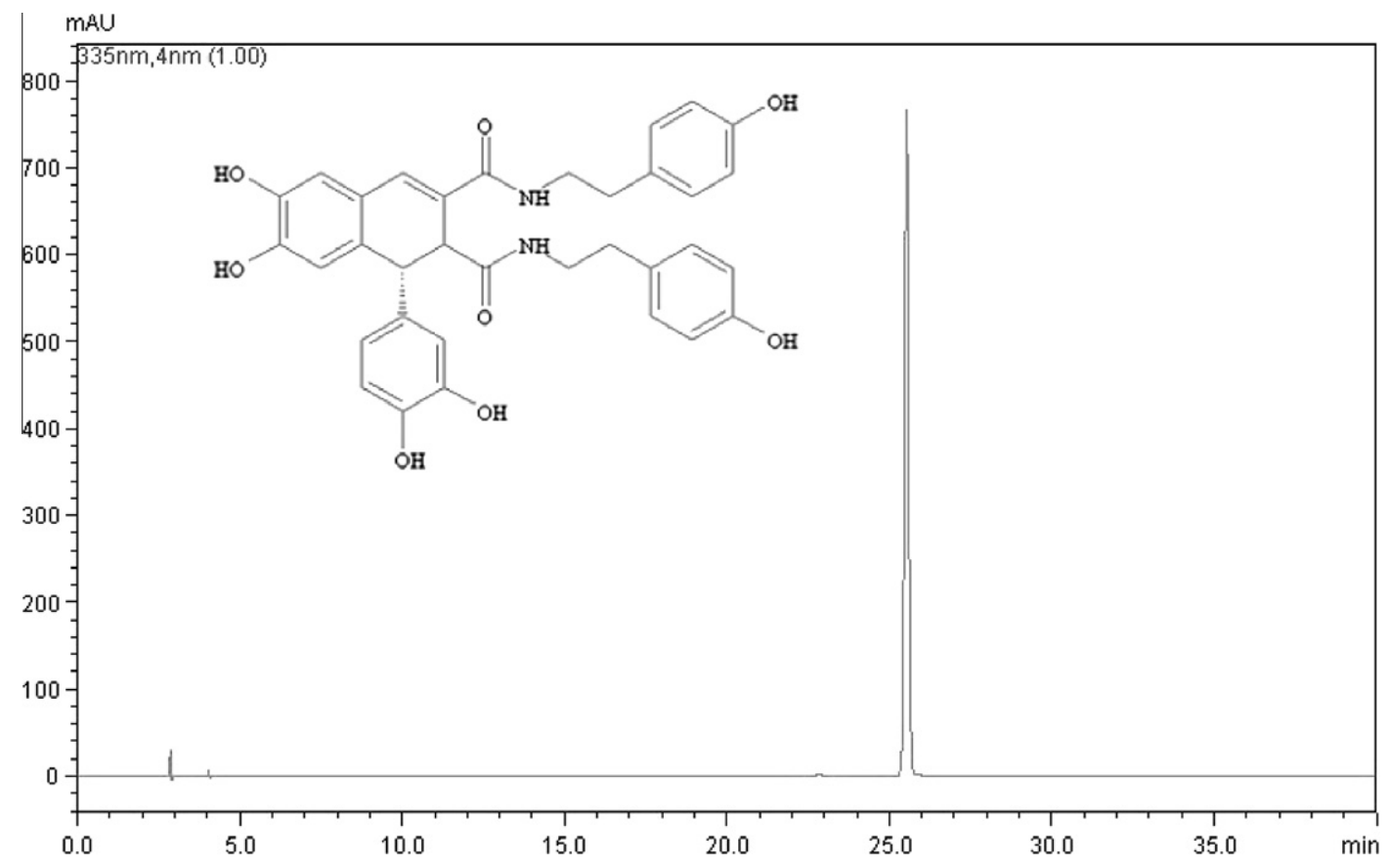

Fig. 1. The structure and HPLC chromatogram of cannabisin B. 


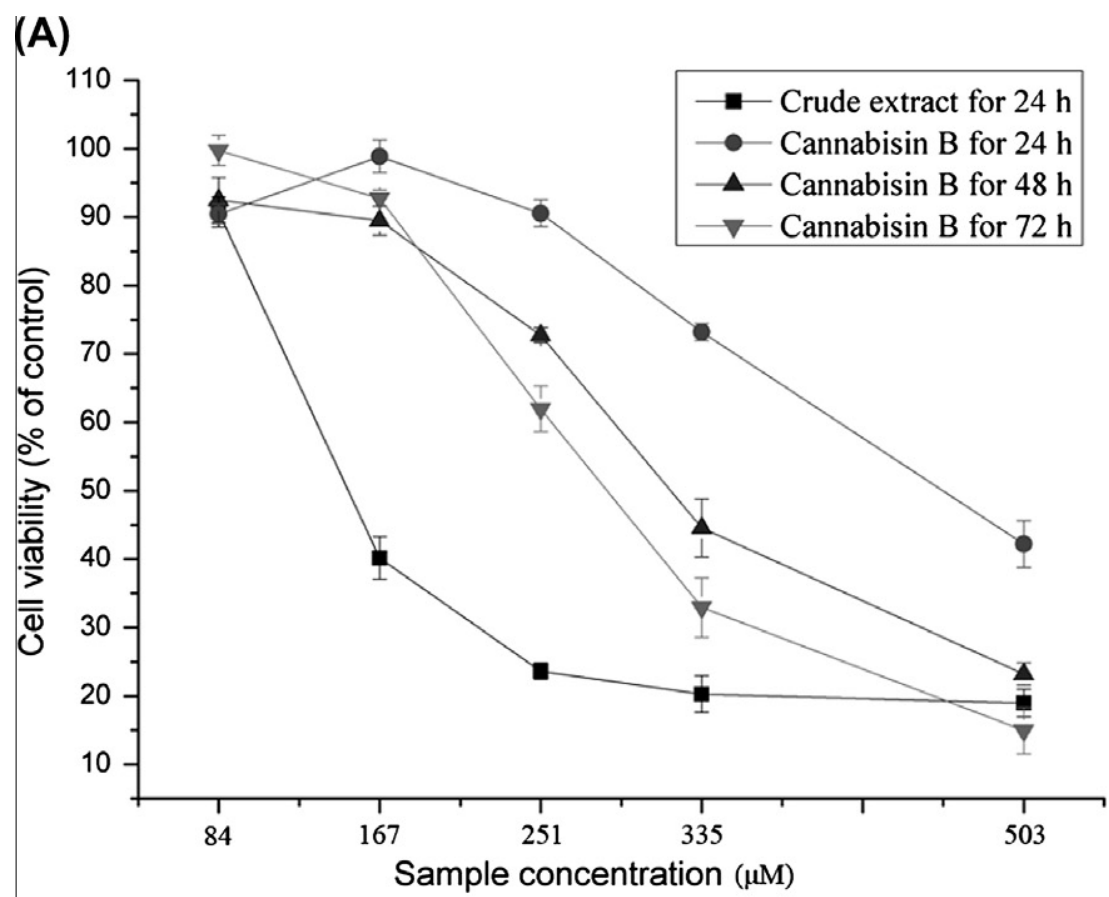

(B)

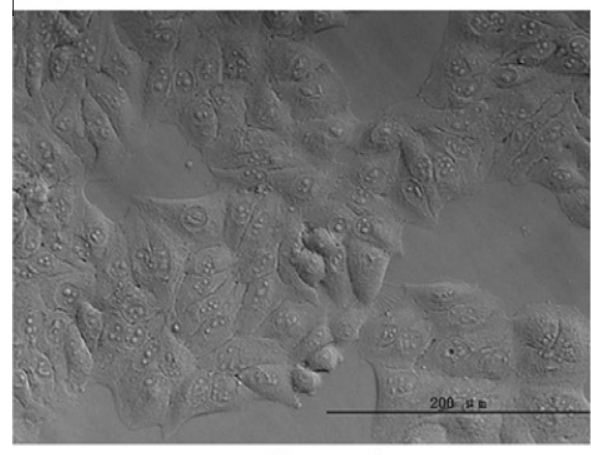

Control

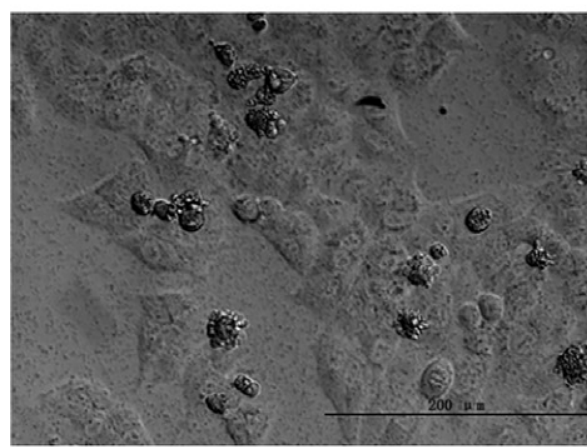

$335 \mu \mathrm{M}$

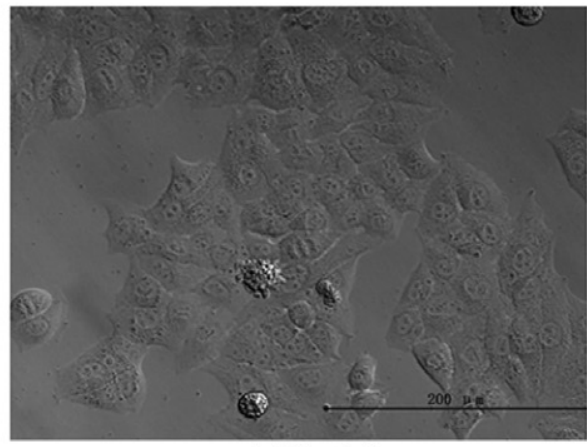

$167 \mu \mathrm{M}$

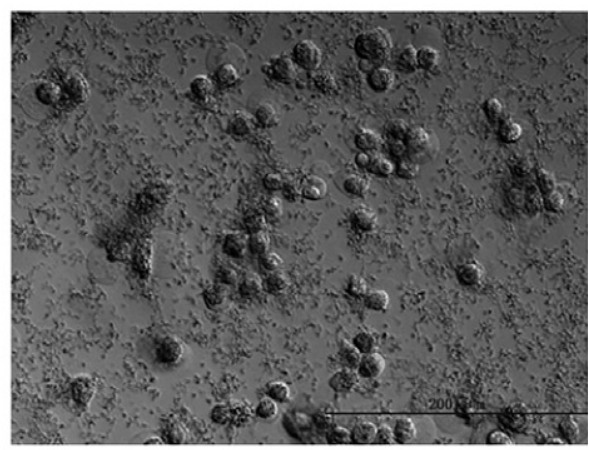

$503 \mu \mathrm{M}$

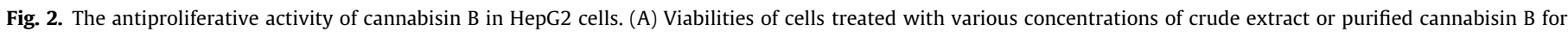
various lengths of time. (B) Images of cells treated with different concentrations of cannabisin B for $24 \mathrm{~h}$.

appearance of phosphatidylserine on the outer side of the plasma membrane (Wu et al., 2004). We previously observed rounding and shrinking in cells treated with cannabisin B and consequently carried out a series of assays to detect apoptosis. Cells stained with Hoechst 33258 were photographed using a fluorescence microscope. As shown in Fig. 4A, the number of condensed nuclei in the cells treated with cannabisin $B$ was greater than that of the control cells, and increased with higher doses of cannabisin B. Although nuclear condensation is a phenomenon that is always associated with apoptotic cells, it is not a definitive indicator of apoptosis.

Flow cytometric analysis of cells stained with annexin V-FITC and propidium iodide (AP) can be used to determine the level of phosphatidylserine turnover from the inner to the outer lipid layer 

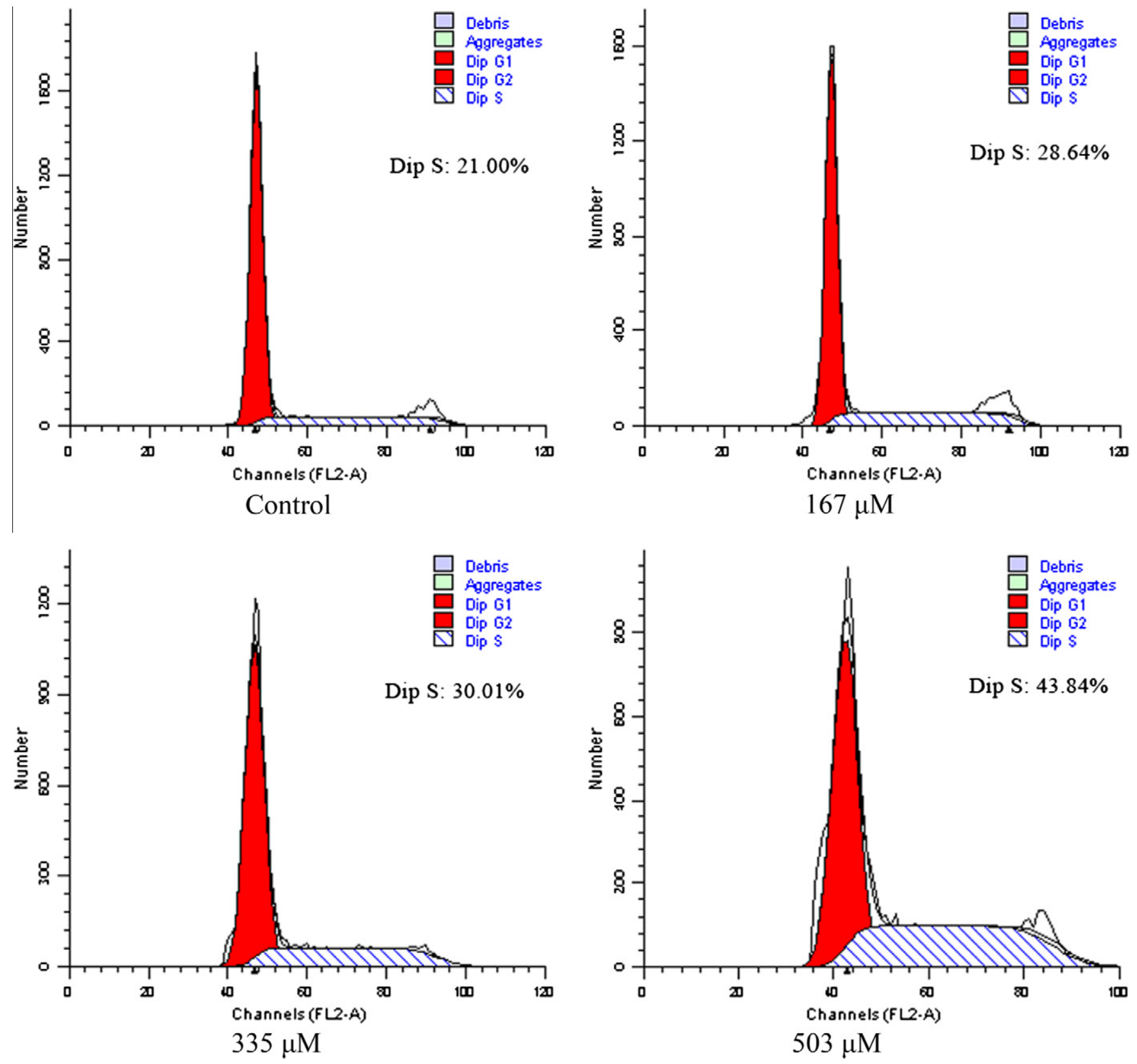

Fig. 3. The effect of cannabisin B on HepG2 cell cycle arrest, as determined by flow cytometry.

of the plasma membrane, which indicates an early stage of apoptosis. The cells that appear in the lower right quadrant are always regarded as apoptotic cells. Fig. 4B showed that there was only a small percentage $(0.3-3.31 \%)$ of treated cells that were defined as apoptotic cells, and that the frequency of this population remained unchanged at higher doses of cannabisin $\mathrm{B}$. The results indicated that cannabisin B (167-503 $\mu \mathrm{M})$ could not induce typical apoptosis in HepG2 cells.

DNA fragmentation, a well-known marker of apoptosis, is indicated by the production of a DNA ladder pattern in an agarose gel following electrophoresis. As shown in Fig. 4C, we did not observe DNA fragmentation in the control samples or the samples treated with different doses of cannabisin B. Though nuclear condensation was observed using the Hoechst 33258 staining assay, apoptosis could not be verified by flow cytometry or DNA ladder detection. The results suggested that the antiproliferative activity of cannabisin B on HepG2 cells cannot be attributed to apoptosis.
3.4. A transient increase in Cannabisin B-induced cell viability results from autophagy

We unexpectedly observed that the cells treated with low concentrations (84-167 $\mu \mathrm{M})$ of cannabisin B exhibited a transient increase in viability compared with the control cells (Fig. 2A), and this increase was not the result of necrosis. Non-apoptotic programmed cell death is predominantly attributed to autophagy (type II programmed cell death), which is a series of biochemical steps through which eukaryotic cells commit suicide by degrading their own cytoplasm and organelles (Reggiori \& Klionsky, 2002). 3-MA, an inhibitor of autophagosomes, is commonly used to block autophagy. Fig. 5A showed that 3-MA could significantly inhibit the increase in viability induced by $167 \mu \mathrm{M}$ cannabisin B; moreover, 3-MA decreased the death rate of cells treated with 335 and $503 \mu \mathrm{M}$ cannabisin B. The cells treated with 3-MA are resistant to the antiproliferative activity of cannabisin $\mathrm{B}$. 
(A)

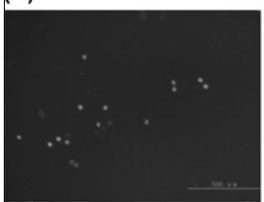

Control

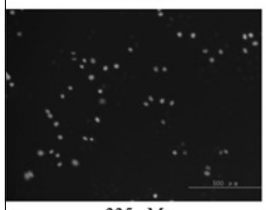

$335 \mu \mathrm{M}$

(B)

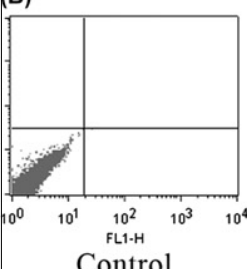

Control

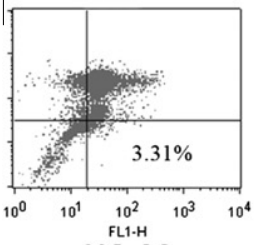

$335 \mu \mathrm{M}$

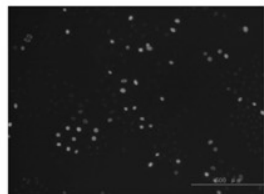

$167 \mu \mathrm{M}$

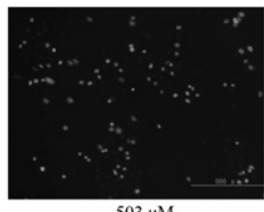

$503 \mu \mathrm{M}$
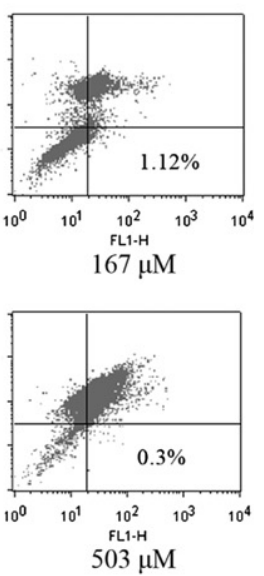

(C)

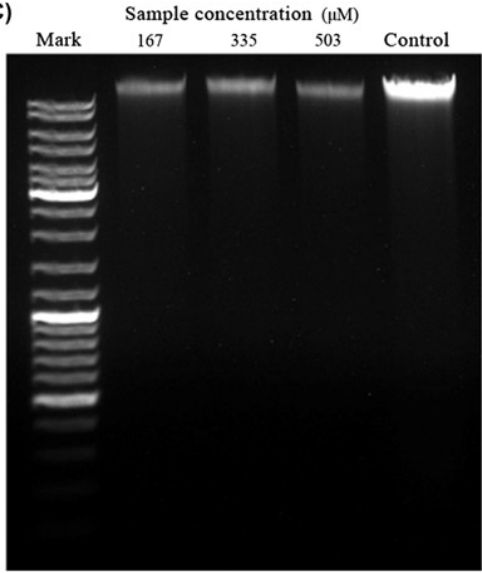

Fig. 4. The detection of apoptosis in HepG2 cells treated with cannabisin B. (A) Fluorescence microscopy was used to capture images of treated cells stained with Hoechst 33258. (B) The flow cytometry plots of cells stained with annexin V-FITC and propidium iodide. (C) DNA fragmentation was determined by agarose gel electrophoresis.

LC3, a group of proteins localized within autophagosome membranes, is regarded as a marker of autophagy (Mizushima et al., 2001). Two forms of cytoplasmic LC3 proteins exist, LC3-I and LC3-II. The amount of LC3-II correlates with the extent of autophagosome formation (Mizushima, Yoshimori, \& Levine, 2010). Western blot analysis (Fig. 5B) indicated that LC3 was expressed in cells treated with cannabisin $B$ alone as well as in cells treated with 3-MA and cannabisin B together. Increased LC3-II expression was only observed in cells treated with $167 \mu \mathrm{M}$ cannabisin B, which also exhibited increased viability compared with the control cells (Fig. 5A).

To determine whether the increase in viability resulted from autophagy induced by cannabisin $\mathrm{B}$, the treated cells were stained
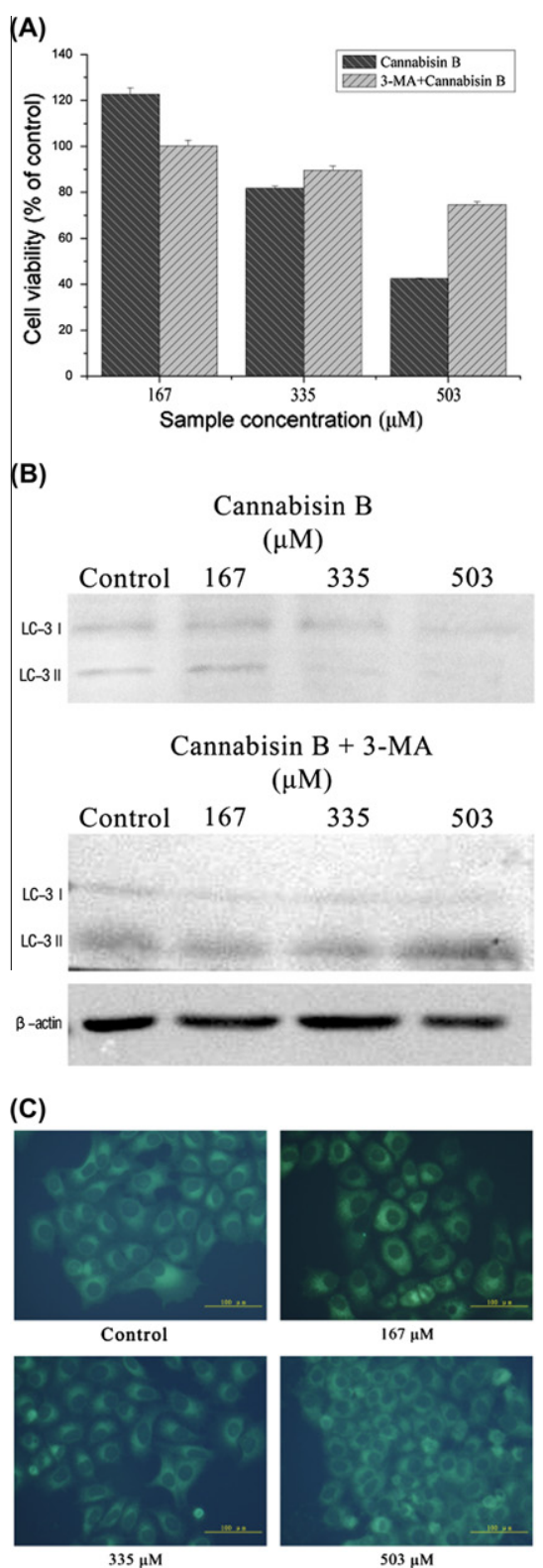

(D)

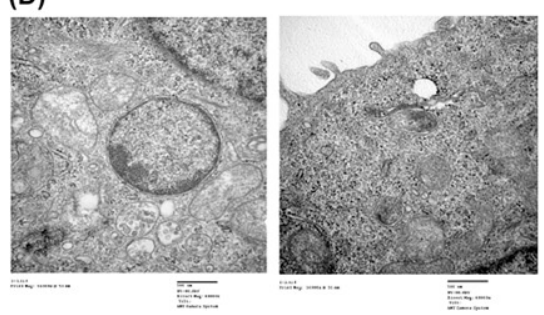

Fig. 5. Cannabisin B induces autophagy in HepG2 cells. Viability (A) and LC3 expression (B) of cells treated with cannabisin $B$ alone or cells pre-treated with 3-MA and then treated with cannabisin B. (C) Fluorescence microscopy was used to capture images of treated cells stained with MDC. (D) The autophagocytic vacuoles examined by transmission electron microscopy.

with MDC and analyzed using a fluorescent microscope. As MDC accumulates in the mature autophagic vascular organelle (AVO), it produces a light spot in the cytoplasm that is indicative of autophagy. As shown in Fig. 5C, the cells treated with $167 \mu \mathrm{M}$ cannabisin B exhibited interspersed staining of AVO, but no significant staining was observed in cells treated with 335 and $503 \mu \mathrm{M}$ 


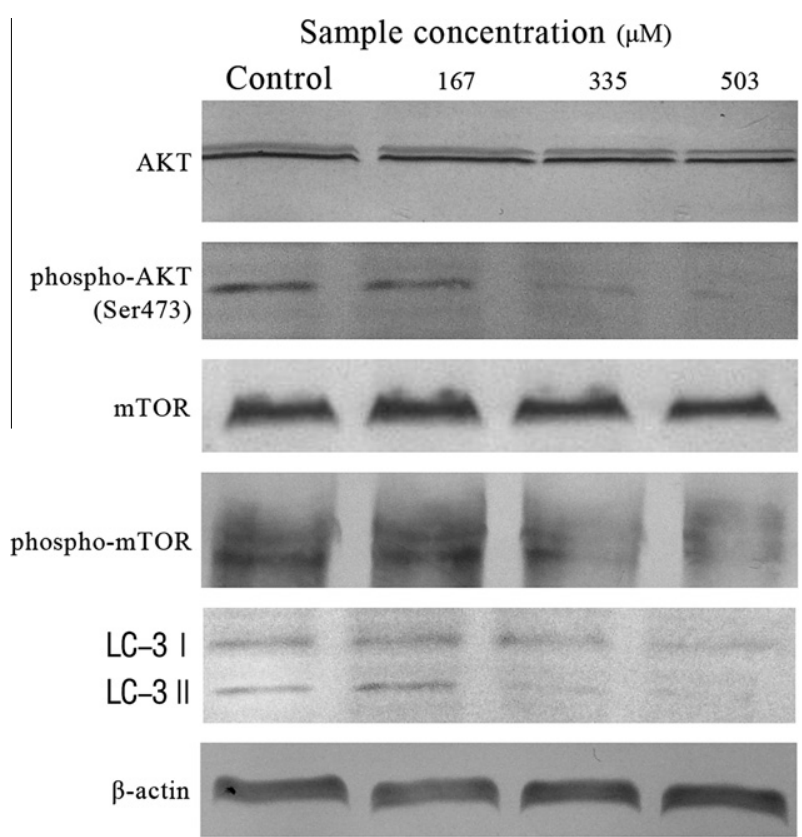

Fig. 6. The effect of cannabisin B on the AKT/mTOR pathway. Total AKT and mTOR, as well as the phosphorylated forms of these proteins, were examined by western blot analysis.

cannabisin B. The existence of an autophagosome was further confirmed by transmission electron microscope (TEM), which is regarded as a gold standard assay for identifying autophagy. In Fig. 5D, cells treated with $167 \mu \mathrm{M}$ cannabisin B exhibit many membrane-bound vesicles that contain organelles and cellular fragments. Collectively, the LC3 expression levels, MDC staining, and TEM demonstrated that cannabisin B transiently promoted the proliferation of HepG2 cells at $167 \mu \mathrm{M}$ and then elicited autophagic death in a dose-dependent manner.

\subsection{Cannabisin B inhibited the AKT/mTOR pathway}

The AKT/mTOR signaling pathway was investigated to determine whether it was involved in cannabisin B-mediated autophagic cell death. Fig. 6 shows the expression levels of ATK, mTOR, and their phosphorylated (activated) forms in cells. Cannabisin B had no impact on total AKT protein but significantly suppressed AKT phosphorylation (Ser 473) in a concentration-dependent manner. Additionally, exposure of HepG2 cells to cannabisin B reduced the levels of the phosphorylated (activated) form of mTOR, a downstream target of AKT, which may have inhibited cell growth and induced autophagy. No effect was observed on total mTOR expression. The results suggested that the autophagy induced by cannabisin B was regulated through the AKT/mTOR pathway.

\section{Discussion}

Cannabisin B is an unusual polyphenol that is found in hempseed, but it has not been identified in any other plants thus far. Cannabisin B possesses considerable antioxidant capacity because of its poly-phenolichydroxyl structure, which was discovered in our previous study (Chen et al., 2012). Numerous studies have demonstrated that polyphenols isolated from plants possess both antioxidant activities and anticancer activities (Alesiani et al., 2010; Carvalho et al., 2010; Kim et al., 2010). In this report, the anticancer properties of cannabisin B were confirmed for the first time, and the results indicate that cannabisin $B$ and the crude extract of hempseed hull can both significantly inhibit the growth of HepG2 cells in a dose-dependent manner. However, the crude extract presents a likely necrosis effect against tumor cells, probably because of other cytotoxic compounds in the extract. Moreover, under this experimental conditions, cannabisin B can arrest cells in the $S$ phase of the cell cycle and induce autophagic cell death, but not typical apoptosis, in HepG2 cells.

Autophagy is often induced upon treatment with various chemotherapeutic agents and is considered an alternative way to kill tumor cells (Coward et al., 2009; Gao et al., 2008). Autophagy begins with the sequestration of cytosolic components, which are ultimately engulfed and digested within double-membraned vacuoles called autophagosomes (Todde et al., 2009). An interesting observation was made during this study: a low concentration of cannabisin B transiently increased cell viability whereas higher concentrations of cannabisin B induced cell death. This observation was ultimately attributed to autophagy after confirming AVO formation by MDC staining, detecting autophagosomes with TEM, and determining that the LC3 marker of autophagy was over-expressed in the cells. Furthermore, 3-MA, an autophagy inhibitor, is able to slow the decline in viability rather than increase or decrease viability sharply. In contrast, the common indicators of apoptosis, such as DNA fragmentation and flow cytometric detection of annexin V-FITC staining, are either nonsignificant or slightly significant and therefore do not support the occurrence of apoptosis in cannabisin B-treated HepG2 cells.

Cell cycle arrest is often observed during programmed cell death. Many reports show that apoptosis always occurs simultaneously with cell cycle arrest in the G2/M phase via interfering with the particular cyclin involved (Lee et al., 2004; Zhao et al., 2011). However, the results of this study indicate that cannabisin B can arrest cells in the $S$ phase of the cell cycle in a dose-dependent manner while simultaneously blocking cell growth. The probable explanation may be that cannabisin B impacts DNA synthesis and/or a cyclin that regulates $S$ phase. Although a positive relationship between $S$ phase arrest and the level of autophagy was identified in this experiment, the mechanism behind this relationship has not been determined and should be investigated in future studies.

Recent studies have confirmed that the inhibition of the AKT/ mTOR pathway is correlated with triggering autophagy in cancer cells (Kim et al., 2006). Activated AKT, which provides key signals to downstream molecules such as mTOR, can inhibit autophagy and promote cell survival (Yap et al., 2008). Our results show that cannabisin B can suppress the phosphorylation of AKT at Ser 473 (the activated form) and consequently inactivate phosphorylated mTOR, though it has no effect on total AKT or mTOR. These findings indicate that cannabisin $\mathrm{B}$ induces autophagic cell death by inhibiting the AKT/mTOR pathway.

In summary, the present study demonstrates (a) that cannabisin $\mathrm{B}$, a bioactive compound from hempseed hull, possesses antiproliferative activity in human hepatocarcinoma HepG2 cells; (b) that cannabisin B arrests cells in the $S$ phase in a dose-dependent manner; and finally, (c) that cannabisin B induces autophagic cell death through the regulation of the AKT/mTOR pathway. The above findings in vitro provide inspiring information of cannabisin $B$ to be utilized against liver tumor. In order to clarify the bioactivity, future research will need to focus on investigating the antiproliferative effects of cannabisin $B$ on other cancer cells as well as the activities in vivo.

\section{References}

Alesiani, D., Canini, A., D’brosca, B., DellaGreca, M., Fiorentino, A., Mastellone, C., et al. (2010). Antioxidant and antiproliferative activities of phytochemicals from Quince (Cydonia vulgaris) peels. Food Chemistry, 118(2), 199-207.

Carvalho, M., Ferreira, P. J., Mendes, V. S., Silva, R., Pereira, J. A., Jerónimo, C., et al. (2010). Human cancer cell antiproliferative and antioxidant activities of Juglans regia L. Food and Chemical Toxicology, 48(1), 441-447. 
T. Chen et al./Food Chemistry 138 (2013) 1034-1041

1041

Chen, T., He, J., Zhang, J., Li, X., Zhang, H., Hao, J., et al. (2012). The isolation and identification of two compounds with predominant radical scavenging activity in hempseed (seed of Cannabis sativa L.). Food Chemistry, 134(2), 1030-1037.

Coward, J., Ambrosini, G., Musi, E., Truman, J. P., Haimovitz-Friedman, A., Allegood, J. C., et al. (2009). Safingol (L-threo-sphinganine) induces autophagy in solid tumor cells through inhibition of PKC and the PI3-kinase pathway. Autophagy, 5(2), 184-193.

De Ioris, M., Brugieres, L., Zimmermann, A., Keeling, J., Brock, P., Maibach, R., et al. (2008). Hepatoblastoma with a low serum alpha-fetoprotein level at diagnosis: The SIOPEL group experience. European Journal of Cancer, 44(4), 545-550.

Gao, M., Yeh, P. Y., Lu, Y. S., Hsu, C. H., Chen, K. F., Lee, W. C., et al. (2008). OSU03012, a novel celecoxib derivative, induces reactive oxygen species-related autophagy in hepatocellular carcinoma. Cancer Research, 68(22), 9348.

He, X., \& Liu, R. H. (2008). Phytochemicals of apple peels: Isolation, structure elucidation, and their antiproliferative and antioxidant activities. Journal of Agricultural and Food Chemistry, 56(21), 9905-9910.

Hirschman, B. A., Pollock, B. H., \& Tomlinson, G. E. (2005). The spectrum of APC mutations in children with hepatoblastoma from familial adenomatous polyposis kindreds. The Journal of Pediatrics, 147(2), 263-266.

Jiang, S., Zu, Y., Fu, Y., Zhang, Y., \& Efferth, T. (2008). Activation of the mitochondriadriven pathway of apoptosis in human PC-3 prostate cancer cells by a novel hydrophilic paclitaxel derivative, 7-xylosyl-10-deacetylpaclitaxel. International Journal of Oncology, 33(1), 103.

Kelekar, A. (2008). Introduction to the review series Autophagy in Higher Eukaryotes-a matter of survival or death. Autophagy, 4(5), 555.

Kim, H., Moon, J. Y., Kim, H., Lee, D.-S., Cho, M., Choi, H.-K., et al. (2010). Antioxidant and antiproliferative activities of mango (Mangifera indica L.) flesh and peel. Food Chemistry, 121(2), 429-436.

Kim, K. W., Mutter, R. W., Cao, C., Albert, J. M., Freeman, M., Hallahan, D. E., et al. (2006). Autophagy for cancer therapy through inhibition of pro-apoptotic proteins and mammalian target of rapamycin signaling. Journal of Biological Chemistry, 281(48), 36883-36890.

Lee, E.-J., Min, H.-Y., Joo Park, H., Chung, H.-J., Kim, S., Nam Han, Y., et al. (2004). G2/ $M$ cell cycle arrest and induction of apoptosis by a stilbenoid, 3,4,5-trimethoxy$4^{\prime}$-bromo-cis-stilbene, in human lung cancer cells. Life Sciences, 75(23), 2829-2839.

Levine, B., Mizushima, N., \& Virgin, H. W. (2011). Autophagy in immunity and inflammation. Nature, 469(7330), 323-335.
Lin, H. M., Tseng, H. C., Wang, C. J., Chyau, C. C., Liao, K. K., Peng, P. L., et al. (2007) Induction of autophagy and apoptosis by the extract of Solanum nigrum Linn in HepG2 cells. Journal of Agricultural and Food Chemistry, 55(9), 3620-3628.

Mizushima, N., \& Levine, B. (2010). Autophagy in mammalian development and differentiation. Nature Cell Biology, 12(9), 823-830.

Mizushima, N., Yamamoto, A., Hatano, M., Kobayashi, Y., Kabeya, Y., Suzuki, K., et al. (2001). Dissection of autophagosome formation using Apg5-deficient mouse embryonic stem cells. The Journal of Cell Biology, 152(4), 657-668.

Mizushima, N., Yoshimori, T., \& Levine, B. (2010). Methods in mammalian autophagy research. Cell, 140(3), 313-326.

Notte, A., Leclere, L., \& Michiels, C. (2011). Autophagy as a mediator of chemotherapy-induced cell death in cancer. Biochemical Pharmacology, 82(5), 427-434.

Prasad, K. N., Xie, H., Hao, J., Yang, B., Qiu, S., Wei, X., et al. (2010). Antioxidant and anticancer activities of 8-hydroxypsoralen isolated from wampee [Clausena lansium (Lour.) Skeels] peel. Food Chemistry, 118(1), 62-66.

Reggiori, F., \& Klionsky, D. J. (2002). Autophagy in the eukaryotic cell. Eukaryotic Cell, $1(1), 11$

Sakakibara, I., Ikeya, Y., Hayashi, K., \& Mitsuhashi, H. (1992). Three phenyldihydronaphthalene lignanamides from fruits of Cannabis sativa. Phytochemistry, 31(9), 3219-3223.

Todde, V., Veenhuis, M., \& van der Klei, I. J. (2009a). Autophagy: Principles and significance in health and disease. Biochimica et Biophysica Acta (BBA) Molecular Basis of Disease, 1792(1), 3-13.

Wu, S.-J., Ng, L.-T., Chen, C.-H., Lin, D.-L., Wang, S.-S., \& Lin, C.-C. (2004). Antihepatoma activity of Physalis angulata and P. peruviana extracts and their effects on apoptosis in human Hep G2 cells. Life Sciences, 74(16), 2061-2073.

Yang, J., Liu, R. H., \& Halim, L. (2009). Antioxidant and antiproliferative activities of common edible nut seeds. LWT - Food Science and Technology, 42(1), 1-8.

Yap, T. A., Garrett, M. D., Walton, M. I., Raynaud, F., de Bono, J. S., \& Workman, P. (2008). Targeting the PI3K-AKT-mTOR pathway: Progress, pitfalls, and promises. Current Opinion in Pharmacology, 8(4), 393-412.

Zhao, Y.-Y., Shen, X., Chao, X., Ho, C. C., Cheng, X.-L., Zhang, Y., et al. (2011). Ergosta$4,6,8(14), 22$-tetraen-3-one induces G2/M cell cycle arrest and apoptosis in human hepatocellular carcinoma HepG2 cells. Biochimica et Biophysica Acta (BBA) - General Subjects, 1810(4), 384-390. 